\title{
Compressed sensing for scanning tunnel microscopy imaging of defects and disorder
}

\author{
Brian E. Lerner $\odot$, Anayeli Flores-Garibay $\odot$, and Benjamin J. Lawrie $\odot$ \\ Materials Science And Technology Division, Oak Ridge National Laboratory, 1 Bethel Valley Rd., Oak Ridge, Tennessee 37831, USA \\ Petro Maksymovych* \\ Center for Nanophase Materials Sciences, Oak Ridge National Laboratory, 1 Bethel Valley Rd., Oak Ridge, Tennessee 37831, USA
}

(Received 31 January 2021; accepted 16 August 2021; published 14 October 2021)

\begin{abstract}
Compressed sensing (CS) is a valuable technique for reconstructing measurements in numerous domains. CS has not yet gained widespread adoption in scanning tunneling microscopy (STM), despite potentially offering the advantages of lower acquisition time and enhanced tolerance to noise. Here we applied a simple CS framework, using a weighted iterative thresholding algorithm for CS reconstruction, to representative high-resolution STM images of superconducting surfaces and adsorbed molecules. We calculated reconstruction diagrams for a range of scanning patterns, sampling densities, and noise intensities, evaluating reconstruction quality for the whole image and chosen defects. Overall, we find that typical STM images can be satisfactorily reconstructed down to $30 \%$ sampling - already a strong improvement. We furthermore outline limitations of this method, such as sampling pattern artifacts, which become particularly pronounced for images with intrinsic long-range disorder, and propose ways to mitigate some of them. Finally, we investigate compressibility of STM images as a measure of intrinsic noise in the image and a precursor to CS reconstruction, enabling a priori estimation of the effectiveness of CS reconstruction with minimal computational cost.
\end{abstract}

DOI: 10.1103/PhysRevResearch.3.043040

\section{INTRODUCTION}

Scanning tunneling microscopy (STM) and spectroscopy (STS) have become indispensable techniques for electronic, structural, and magnetic characterization of surfaces with atomic resolution. STM has enabled investigations of broken symmetry and vortex interactions in superconductors [1,2], band-structure mapping of quantum materials [3], and even manipulation of single atoms [4-6].

However, small tunneling currents limit the rate of current measurement to the millisecond timescale, so STM measurements are characterized by comparatively long measurement times [7]. This limitation becomes apparent in experiments that seek to probe extended surface areas, seek rare events such as low density defects, and want to strike a balance between high-resolution measurements in real space and energy resolution. In such cases, the ability to accurately reconstruct the underlying periodic and defect structures of nanoscale samples with reduced measurement times is highly desirable.

Compressed sensing (CS) shows potential for meeting this demand. CS is based on the notion that if a basis set can be found where the signal is sparse (and as a corollary

\footnotetext{
*maksymovychp@ornl.gov

Published by the American Physical Society under the terms of the Creative Commons Attribution 4.0 International license. Further distribution of this work must maintain attribution to the author(s) and the published article's title, journal citation, and DOI.
}

the signal is compressible in that basis), accurate reconstruction is possible using fewer measurements than required by the Shannon-Nyquist sampling theorem. CS has been successfully employed for diverse applications, including radio interferometry [8], nuclear magnetic resonance of protein structure $[9,10]$, recovery of correlations of entangled photon pairs [11,12], medical imaging [13,14], and many more.

An image is compressible by virtue of its sparsity in a transform domain. Most images in the natural world have a sparse representation in a plane-wave or wavelet basis, including those generated by scanning microscopies. Indeed, CS has been successfully implemented in scanning electron [15], atomic force [16], and piezoresponse force microscopy [17], and quasiparticle interference imaging by STS [7,18]. However, a detailed understanding of the potential of CS for STM has yet to be developed, particularly with respect to imaging defects and disorder.

In this paper, we explore the parameter space of a simple CS framework in the context of representative STM images from surfaces of superconductors and single molecule layers (introduced in Sec. II). Our specific focus is to emphasize the quality of reconstruction around defects and as a function of added noise. In Secs. III and IV, the basic methodology of CS is laid out and the framework is described. Using a soft weighted iterative thresholding (SWIT) algorithm of practical computational complexity, we performed reconstructions across variable noise perturbation intensities and sampling densities. These reconstructions are evaluated for structural similarity index measure (SSIM) and mean squared error (MSE) and are used to build reconstruction 

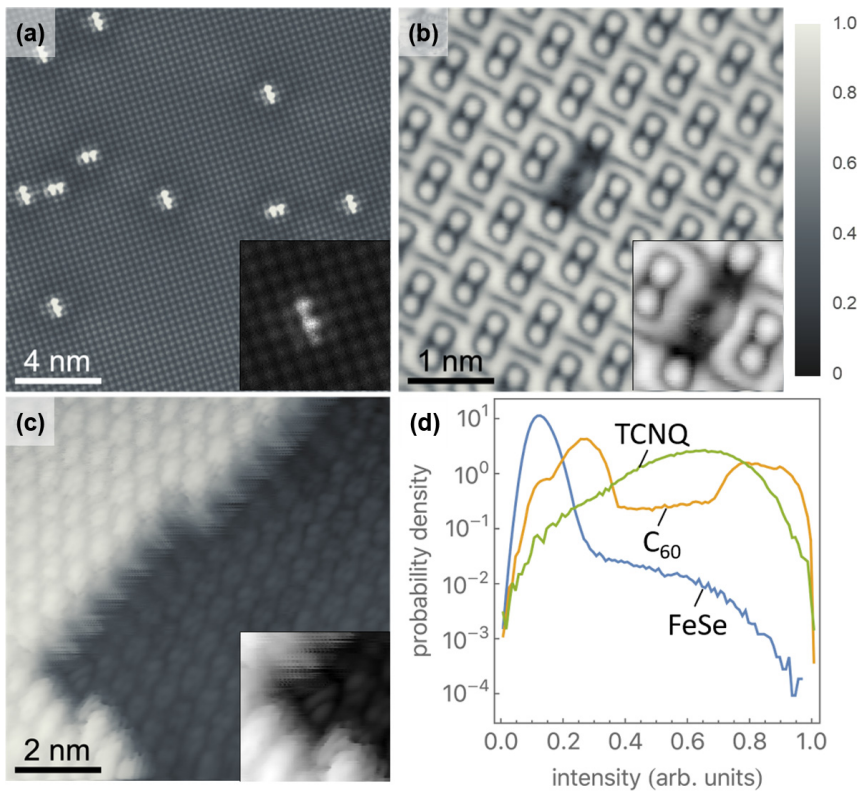

FIG. 1. STM images of FeSe (a), molecular film of TCNQ/Ag(111) (b), and that of $\mathrm{C}_{60} / \operatorname{Ag}(111)$ (c), with representative defects magnified in each inset. (d) The distribution of normalized constant-current STM height for each image.

diagrams in Sec. V. Our results reveal that accurate reconstruction can be obtained at sampling densities as low as 20-30\% for images with both point and extended nanoscale defects-i.e., with up to fivefold compression. We also note artifacts arising in the reconstructions and detail ways of mitigating these deviations through proper algorithm configuration. To effectively apply CS in practice, it is very helpful to understand what types of images can be effectively reconstructed. In Sec. V, we show that compressibility is an effective measure of noise in the STM images and a necessary, albeit not sufficient, criterion for effective CS reconstruction.

\section{EXPERIMENTAL DATA}

We applied CS to representative STM images of a cleaved 100-surface of FeSe superconductor with Se vacancy defects [19] [Fig. 1(a)] and two kinds of adsorbed molecular layers$\mathrm{C}_{60}$ on $\mathrm{Ag}(111)$ [Fig. 1(c)] and TCNQ (tetracyanoquinodimethane) on highly oriented pyrolitic graphite [Fig. 1(b)]. Each of the sample images have different sizes, lattice structures, and point or extended defects. Moreover, as seen in Fig. 1(d), the images represent three kinds of intensity distribution, centered on low values corresponding to the atomic lattice in the case of $\mathrm{FeSe}$, a broader and more uniform distribution in the case of TCNQ, and a distinctly bimodal distribution for $\mathrm{C}_{60}$, owing to a single atomic step of the underlying substrate and therefore two distinct levels of intensity. Each of these images were obtained at a similar spatial resolution on the atomic scale, though they vary in their size. In terms of pixels, TCNQ is $256 \times 256, \mathrm{C}_{60}$ is $512 \times 512$, and FeSe is $1024 \times 1024$.

\section{CS BASICS}

Sparsity regularization is a common approach to impose constraints on undefined optimization problems [20], which gave rise to CS methodology in the mid-2000s [21,22]. CS is designed to reconstruct a signal $x \in \mathbb{R}^{n \times 1}$ from samples $y \in \mathbb{R}^{m \times 1}$, where typically $m \ll n$. Successful reconstruction is possible when $x$ has a sparse representation $\alpha \in \mathbb{R}^{n \times 1}$, i.e., in some basis the number of significant coefficients $k$ in $\alpha$ is small compared to $n$. A CS reconstruction algorithm computes $\alpha$. Once obtained, $x$ is recovered using the basis transform $\Psi \in \mathbb{R}^{n \times n}:$

$$
x=\Psi \alpha .
$$

The sampling process has a matrix representation $\Phi \in \mathbb{R}^{m \times n}$ constructed by stacking each measurement vector:

$$
\Phi x=y
$$

Using Eq. (1) to substitute for $x$ in Eq. (2) and setting $A=$ $\Phi \Psi$, we have

$$
A \alpha=y .
$$

CS provides a solution $\alpha$ for this undetermined system of equations by minimizing the sparsity of $\alpha$ under the constraints of Eq. (3), expressed as

$$
\min \|\alpha\|_{\ell_{0}} \quad \text { s.t. } \quad A \alpha=y .
$$

While this approach provides an exact solution, $\ell_{0}$ minimization is a combinatorial optimization problem that is computationally expensive, and intractably so for large signals [22]. Fortunately, the $\ell_{1}$ norm can be substituted to convert the problem into one of convex optimization, where for most inputs, $\alpha$ is recovered exactly [22].

\section{FRAMEWORK}

The CS framework can utilize a variety of (1) sampling matrices $\Phi$, (2) transform matrices $\Psi$, and (3) reconstruction algorithms. $\Psi$ should necessarily be chosen to ensure sparsity in the transform domain but it should also be incoherent with $\Phi$. Incoherence, which along with sparsity is a guiding assumption underpinning CS, dictates that $\Phi$ should be spread out (i.e., nonsparse) in the transform basis [23]. The algorithm minimizes the sparsity in $\alpha$ while remaining correlated to the measurements $y$ [Eq. (3)]. In our implementation of a CS framework, we use rotated line and Lissajous trajectories as the sampling patterns, the discrete cosine transform (DCT) as a basis transform, and a SWIT reconstruction algorithm. The elements of this framework, with special regard to their applicability for STM, are discussed in the following.

\section{A. Transform matrix}

STM images often exhibit a large amount of order and are generally smooth (i.e., differentiable in the absence of noise). As a result, the images lend themselves to sparsity in the DCT basis (Fig. 2). The DCT transform matrix also has the advantage of being maximally incoherent with point sampling matrices [23] and has a fast matrix implementation [24]. This transform has been utilized in previous applications of CS [25-27] and has historically been used for JPEG compression 

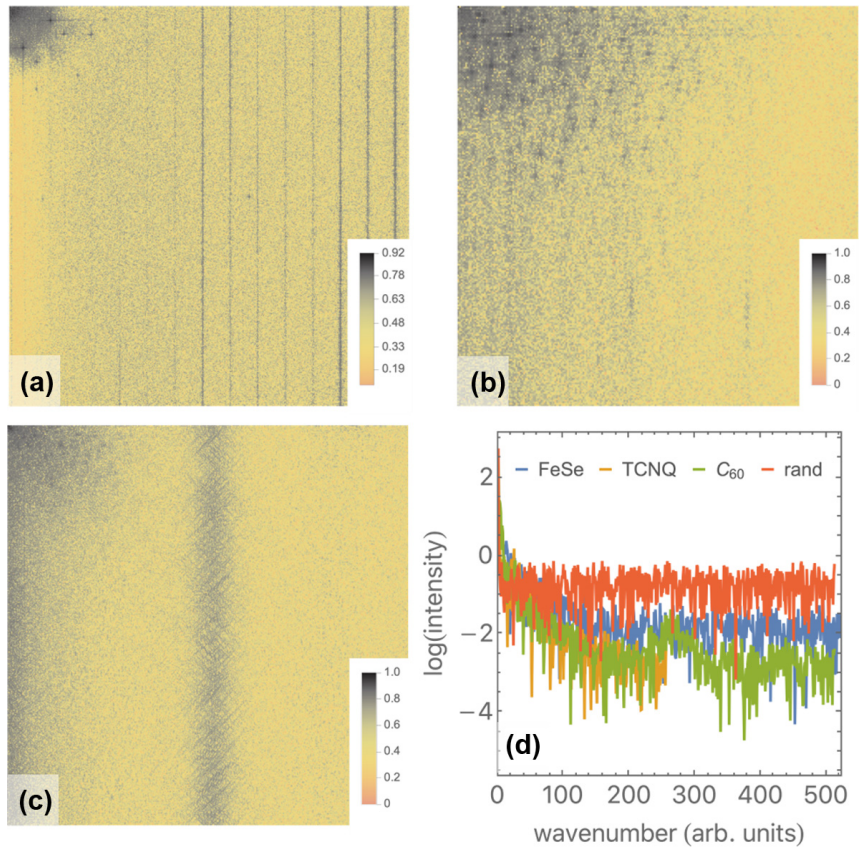

FIG. 2. Discrete cosine transform of the STM images in Fig. 1, correspondingly for (a) FeSe, (b) TCNQ, and (c) $\mathrm{C}_{60}$. (d) The intensity of the diagonal coefficients for each DCT, as well as the diagonal of the DCT for an array of random Gaussian noise, which reveals sparsity of STM images.

[28]. The discrete wavelet transform is another commonly used dictionary in CS, thought it works most efficiently with dense sampling matrices with random entries like those used for single-pixel imaging and is less incoherent than DCT for point sampling matrices [24].

\section{B. Sampling matrix}

When scanning a surface, it is conventional to use a raster scan, resulting in an evenly sampled grid of point measurements. The speed of the probe and the sampling frequency are set based on the demands of the experiment. While the design of the sampling matrix $\Phi$ in other CS applications is often flexible (programmable with a spatial light modulator for optical CS applications, for instance [12]), we are constrained to sampling along the continuous path of the probe. Here, since we are concerned with the algorithmic aspects of the reconstruction, we chose to use pre-existing STM images and resample them with the rotated line [Figs. 3(a) and 3(b)] and smooth Lissajous [Figs. 3(d) and 3(e)] patterns which make the methods more compatible with fast scanning. The sampling can furthermore be randomized along the sampling path, but we have not seen a significant impact from such randomization.

\section{Reconstruction algorithm}

As noted, solving Eq. (4) is too expensive to solve with traditional means. The most common approach for relaxing this constraint is to instead minimize the $L_{1}$ norm, allowing for a family of problems that can be solved through convex optimization algorithms such as LASSO and basis pursuit

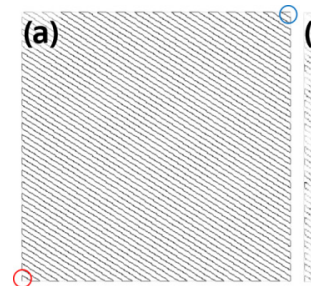

(b)

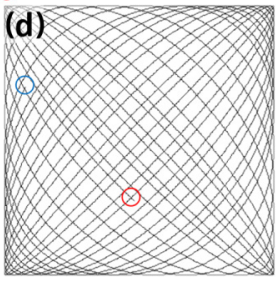

(e)

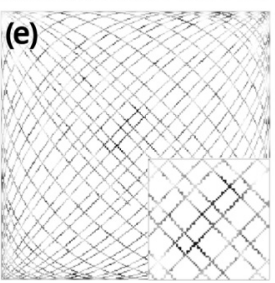

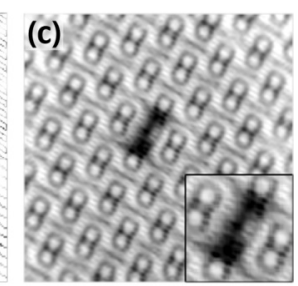

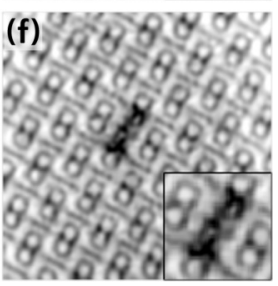

FIG. 3. The path of the rotated line pattern is shown in (a), with simulated start and end points denoted by green and red circles. Despite sparse sampling of the image (b), very good CS reconstruction is achieved (c). The same process is also shown for Lissajous trajectory (d)-(f). Reconstructions in this figure performed for $20 \%$ sampling density and 100 iterations.

denoising. Greedy iterative algorithms, which seek a solution by making locally optimal decisions, offer an alternative approach. While they tend to be less effective than convex optimization methods [29], they have been shown to be just as effective in certain cases and, importantly, offer reduced computational complexity and robustness to noise. A subclass of this family are the iterative thresholding [30] algorithms, which have an iterative form of

$$
\alpha^{i}=\eta_{t}\left(\alpha^{i-1}+\kappa A^{T}(y-A \alpha)\right)
$$

The nonlinear thresholding function $\eta_{t}$ performs elementwise operations on a vector input and $\kappa$ is a relaxation parameter that allows the algorithm to converge. Two often implemented variants of $\eta_{t}$ are the hard [31] and soft [32] threshold operators:

$$
\begin{aligned}
\eta_{t}^{h}(x) & = \begin{cases}x, & |x|>t \\
0, & |x| \leqslant t,\end{cases} \\
\eta_{t}^{s}(x) & = \begin{cases}\operatorname{sgn}(x)(|x|-t), & |x|>t \\
0, & |x| \leqslant t .\end{cases}
\end{aligned}
$$

The threshold $t$ can be fixed, which allows the sparsity of $\alpha$ to vary. Alternatively, the desired sparsity of the solution can be specified, allowing $t$ to fluctuate as required in each iteration. Studies applying CS to atomic force microscopy images show better reconstruction using the soft variant [24], which can be further improved by using a soft weighted operator [16] to apply weights $w \in \mathbb{R}^{n \times 1}$, where each weight $w_{i}$ is associated with a coefficient $\alpha_{i}$ :

$$
\eta_{t}^{w s}(x)= \begin{cases}\frac{1}{w_{i}} \operatorname{sgn}(x)\left(\left|w_{i} x\right|-t\right), & \left|w_{i} x\right|>t \\ 0, & \left|w_{i} x\right| \leqslant t\end{cases}
$$

By tuning the weights to model expected DCT dispersion, weighted iterative thresholding algorithms tend to outperform their nonweighted counterparts [16]. In our experiments, we utilize $\eta_{t}^{w s}$ as part of a SWIT implemented in PYTHON [16] as 
follows:

$$
\begin{aligned}
& \begin{array}{l}
\alpha=0 \\
r=y
\end{array} \\
& \text { for } \quad \mathrm{i} \text { in } I_{\max }: \\
& \\
& \quad c=A^{T} r \\
& \alpha=\eta_{t}^{w s}(\alpha+\kappa c) \\
& r=y-A \alpha \\
& \text { if }\|r\|_{\ell_{2}}<\epsilon\|y\|_{\ell_{2}} \text { : } \\
& \quad \text { break }
\end{aligned}
$$

For our reconstructions, we initialize $\alpha$ to 0 (though if prior information is known about the image, $\alpha$ can be initialized to an educated guess), and the stopping condition is defined by the residual-measurement ratio. Unless otherwise noted, we specify a 5\% sparsity level, allowing the threshold $t$ to fluctuate. Based on recommendations in the literature [16,29,33], we set $\kappa=0.6$ and $\epsilon=0.001$. The initial weights of the soft thresholding function $\eta_{t}^{w s}$ used in these reconstructions are adopted from a Gaussian model of DCT structure in Ref. [16], which was used to successfully reconstruct atomic force microscopy images. The maximum number of iterations $I_{\max }$ was set to 100 due to computational considerations, though in our experiment we found that reconstruction tends to improve up to around 300 iterations - and sometimes many more-before plateauing.

\section{Quality assessment}

Using a CS framework composed of a DCT transform in combination with the noted sampling patterns and implementation of the SWIT algorithm, we evaluated the algorithm while systematically varying the noise intensity $\delta$ and sampling density $\rho$ to understand the limits of the reconstruction. While iterative thresholding algorithms are noted for being noise robust [34], little investigation has been carried out to confirm this for reconstruction of STM images. To test this, we generated $1 / f$ noise and added it to values along the simulated measurement path so as to mimic varying noise levels during measurement. The noise perturbation scale for each image was normalized to range from $0.1-1$ of the highest-peak full width at half maximum (FWHM) in the intensity histogram of the image [Fig. 1(d)]. Rotated line and Lissajous sampling patterns were implemented across $\rho$ from $0.02-0.5$. The patterns used here were generated using magni [33], a CS PYTHON package for atomic force microscopy.

For each reconstruction in this $\delta$ - $\rho$ parameter space, the quality of the reconstructed image was evaluated for SSIM and MSE. SSIM was calculated using the default implementation in SCIKIT-IMAGE [35], itself adapted from Ref. [36]. The MSE is calculated in the standard way,

$$
\frac{1}{N} \sum(\chi-x)^{2}
$$

where $N$ is the number of pixels, $x$ is the reconstructed image, and $\chi$ is the base image.
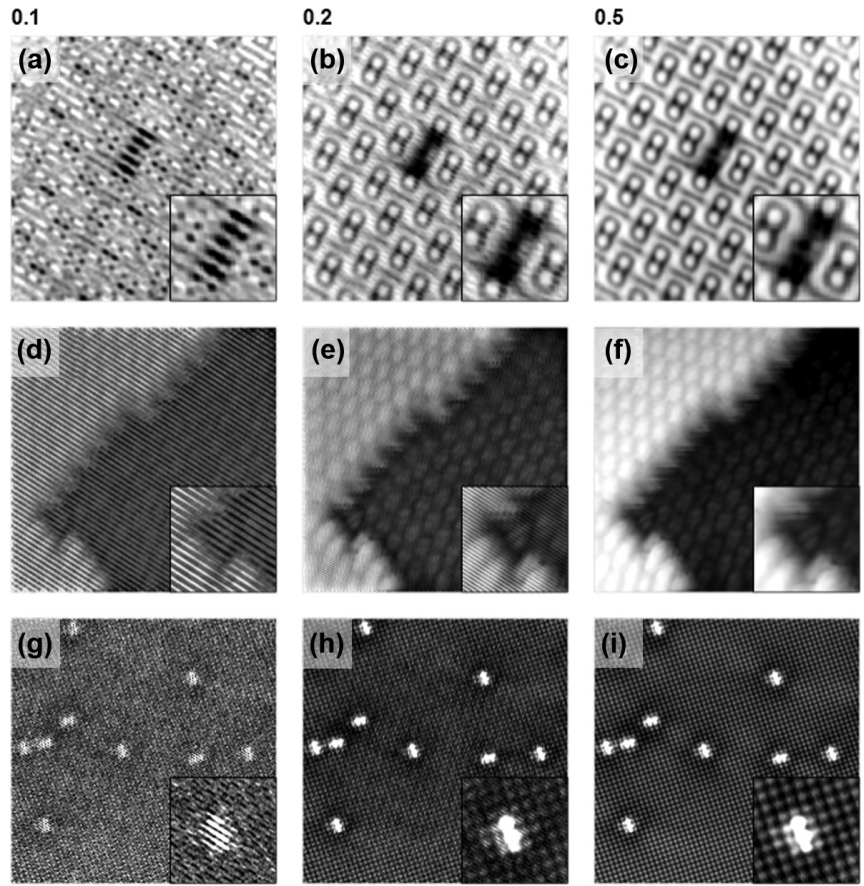

FIG. 4. Reconstructed images for ten-, five-, and twofold undersampling for TCNQ (a)-(c), $\mathrm{C}_{60}$ (d)-(f), and FeSe (g)-(i), with magnified defects in insets. All reconstructions were performed for 100 iterations using the rotated line sampling pattern.

\section{RESULTS}

Our first observation is that CS is generally very good at reconstructing STM images even at a sampling density as low as $20 \%$ of the original image [Figs. 3(c), 3(f) and 4]. To ascertain that this conclusion applies not only to spatial order in the images but also to defect sites, we have identified defects using scale-space methods for detection of protrusions (using Laplacian of Gaussian filter [37]), and then built local masks, comparing reconstruction in that local region. As seen in the insets of Fig. 4, single vacancies in FeSe and extended defects in the TCNQ film (missing molecules) do indeed reconstruct well for the rotated line pattern. In fact, at 50\% sampling density, the reconstructed defects are nearly indistinguishable from their unsampled counterparts.

The rotated line $\delta$ - $\rho$ reconstruction diagrams (Fig. 5) demonstrate our method's robustness to moderate $1 / f$ noise. All reconstructions have high SSIM above sampling density $\rho \approx 30 \%$, which only begins to degrade at noise perturbations of 0.4 for TCNQ and 0.8 for FeSe. While high-noise distortions are apparent in the reconstructions of TCNQ [Fig. 5(a)] and FeSe [Fig. 5(c)], the simplicity of FeSe's vacancies and the regularity of its lattice likely lead to smoother SSIM falloff at high noise. The transition for $\mathrm{C}_{60}$, in stark contrast, exhibits a much smaller sensitivity to added noise [Fig. 5(e)]. $\mathrm{C}_{60}$ also exhibits a sharp transition from low to high SSIM (poor to good reconstruction) at sampling density around 30\%, which exceeds the transition point of the other samples by $10-20 \%$. Visual examination of the reconstructions reveals the presence of sampling pattern artifacts at low SSIM which disappear after the transition line [Fig. 5(h)]. The reasons for this deviation will be discussed further below. 

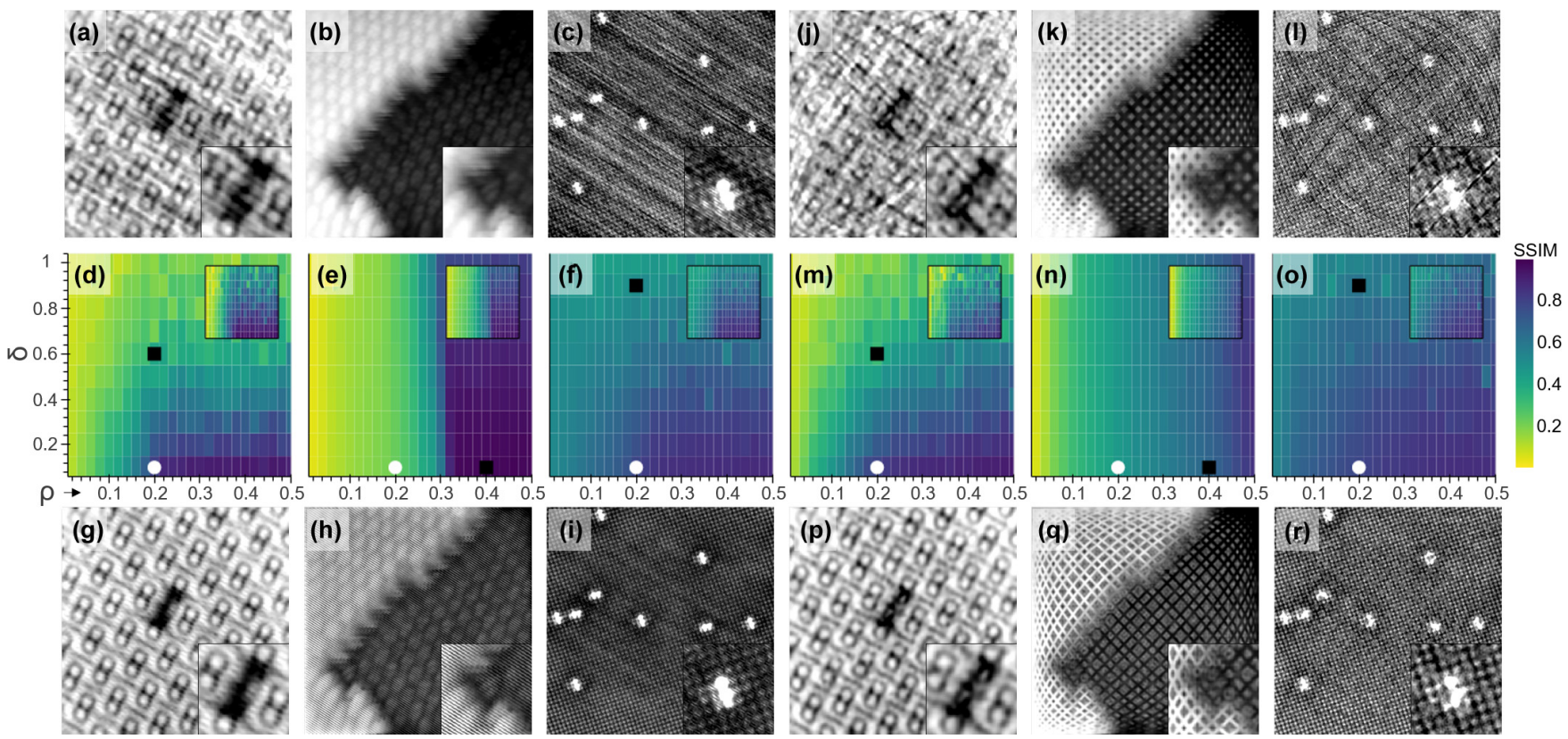

FIG. 5. Reconstruction diagrams for TCNQ, $\mathrm{C}_{60}$ and FeSe for the rotated line (d)-(f) and Lissajous (m)-(o) patterns, with relevant reconstructions shown above and below the diagram for each sample. The diagram plots SSIM of the reconstructed image versus intensity of noise perturbation intensity $(\delta)$ and sampling density $(\rho)$. The parameters used for the reconstructions in the top row are marked by black squares in the respective diagrams; the bottom row parameters are marked by white circles.

In contrast to the rotated line pattern, the Lissajous pattern suffers from reduced sampling density toward the center of the image and larger gaps throughout. This leads to sampling artifacts [Figs. 5(k) and 5(q)] and difficulties in reconstructing small-scale and centrally located image structures. As a result, the rotated line achieves a higher peak SSIM than Lissajous, though the reconstruction diagrams for the latter show greater noise robustness in the moderate-to-high noise regime. Given that CS is predicated on the principle of compression, we explored the extent to which our CS results correlate to image compressibility for typical STM images [Fig. 6(a)]. For comparison, we included simulated images, a pseudorandom image sampled from a uniform distribution [Fig. 6(b)], and an image of an ordered lattice [Fig. 6(c)]. We evaluated compressibility via a sequence of DCT transform, elimination of select coefficients, a back-transform, and, finally, calculation of MSE relative to the uncompressed image. The pseudorandom image displays the highest MSE across compression sizes [Fig. 6(a)], i.e., it is most incompressible, while the ordered lattice is the most compressible and, correspondingly, displays the slowest and fastest drop of MSE as a function of compressed size. The STM images fall between these two extremes. Intriguingly, there is a very significant difference between individual experimental images, which actually goes against the trend that may be inferred from the visual inspection of the original data in Fig. 1. $\mathrm{C}_{60}$, not TCNQ nor $\mathrm{FeSe}$, is the most compressible image, while FeSe is notably less compressible than either TCNQ or $\mathrm{C}_{60}$. The difference in compressibility stems from the signal-to-noise ratio that characterizes these images. To ascertain that this is the case, in Fig. 6(d) we plot compressibility of the TCNQ image as a function of strength of added Gaussian noise (measured as a fraction of the largest signal in the image). The com- pressibility curve very clearly shifts up, traversing nearly the whole range of compressibility in Fig. 6(a) and eventually becoming equivalent to noise. We note that the STM images

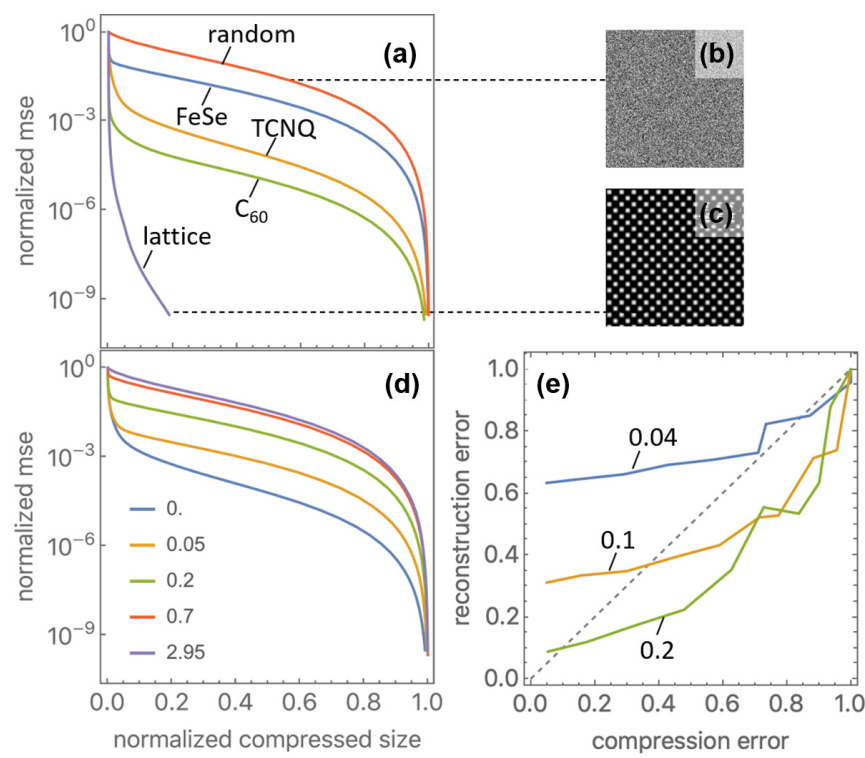

FIG. 6. Compressibility of STM images (a), along with random noise (b) and an ordered lattice (c) via DCT transform. The MSE curves as a function of compressed size were normalized against the peak value for each curve. Compressibility of TCNQ for varying levels of Gaussian noise applied to TCNQ (d) (curves increase in noise level from bottom to top). (e) Parametric plot of compression error versus CS reconstruction error with added Gaussian noise as a parameter. The curves were calculated for three values of compression $(0.04,0.1$, and 0.2$)$. 


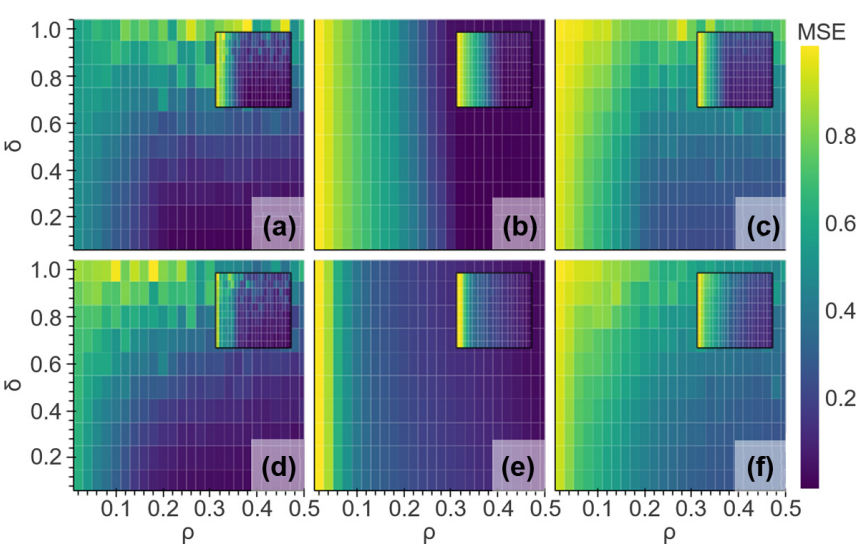

FIG. 7. Reconstruction diagrams of CS reconstruction of TCNQ [(a), (d)], $\mathrm{C}_{60}[(\mathrm{~b}),(\mathrm{e})]$, and FeSe [(c), (f)] calculated for rotated line (top row) and Lissajous (bottom row) sampling patterns using MSE quality metric. Insets show diagrams for defects. All reconstruction diagrams have been normalized to their respective maximum MSE.

used here were all acquired on different days, with different physical tips and different instrument conditions. The ability to evaluate the STM image via compressibility appears to be a valuable measure of the data quality and experimental results.

We now show that compressibility also generally correlates with the performance of CS. In Fig. 6(e), we parametrically plot the normalized CS reconstruction error versus the normalized DCT compression error, with noise as a parameter. For fivefold compression (20\% sampling, green curve), the correlation is reasonably good, which confirms our notion. However, for larger compressions at $10 \%$ and $4 \%$ sampling, CS systematically produces higher errors than obtained by DCT compression, which reduces the correlation between the two techniques. We speculate that these deviations are partly due to CS being sensitive to the compatibility of sampling and transform matrices with each other and with the image, and possibly due to finer details of the algorithm and its hyperparameters.

A striking disparity, however, appears for $\mathrm{C}_{60}$, which is the most compressible of the typical STM images [Fig. 6(a)] but requires the highest sampling density to achieve a quality CS reconstruction [Fig. 5(e)]. Interestingly, $\mathrm{C}_{60}$ generally performs the best with regard to both the SSIM (Fig. 5) and MSE (Fig. 7) reconstruction diagrams, followed by TCNQ and then FeSe. So, the best reconstruction here correlates to the superior compressibility of the $\mathrm{C}_{60}$ image. But below the transition line, $\mathrm{C}_{60}$ performs worst for both metrics. Resolving this puzzle depends on an understanding of how and when sampling pattern artifacts appear. We have found that artifacts, including those in Fig. 5(h), can be removed by properly configuring the SWIT algorithm. Small disturbances can be removed by increasing the number of iterations, but more prominent artifacts require increased iterations and/or specialized setup of the threshold function [Eq. (8)] as explained below.

In each iteration of the SWIT, the threshold function weights each coefficient using a DCT model [Fig. 8(a)] and, based on a specified threshold ratio, keeps a certain number of

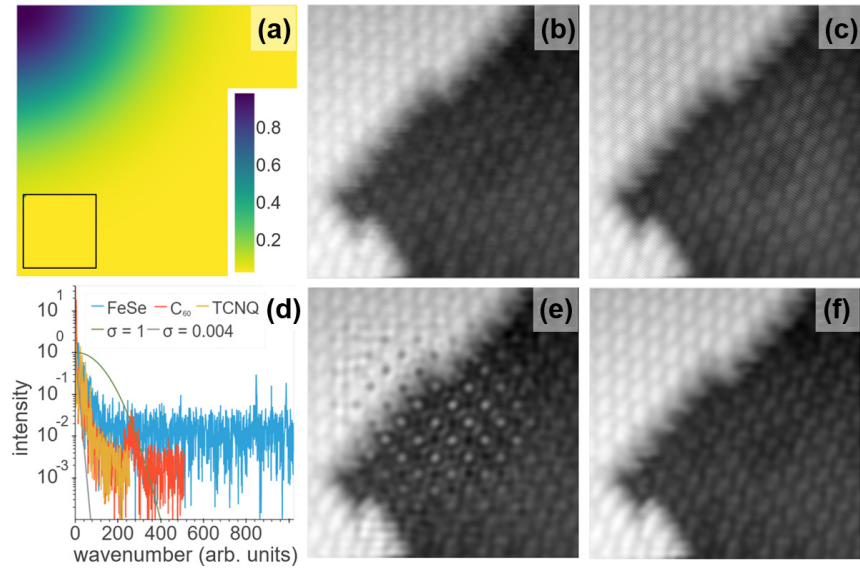

FIG. 8. (a) A wide-variance DCT weight model used to reconstruct $\mathrm{C}_{60}$ for Lissajous (b) and rotated line (c) patterns using 300 iterations and a $1 \%$ threshold on the number of nonzero coefficients. The low-frequency corner snippet of a low-variance model is shown in the inset of (a), and the diagonal coefficients for both models and each sample DCT are displayed in (d). Reconstructions using the low-variance model are shown for Lissajous (e) and rotated line (f).

coefficients while setting the rest to 0 . We show that setting the threshold ratio to $1 \%$ instead of 5\%, running for 300 iterations, and minimizing the variance in the weight model, the artifacts can be removed from $\mathrm{C}_{60}$. Reconstruction with the Lissajous pattern was more responsive [Figs. 8(b) and 8(e)] to the wider DCT-model variance used to compute Figs. 5 and 7, whereas the rotated line reconstructions improved [Figs. 8(c) and 8(f)] only with severely minimized variance [Fig. 8(a), inset] which effectively imposed a very sharp cutoff of the low-pass filter [Fig. 8(d)].

To determine the best thresholding function parameters, we evaluated $\mathrm{C}_{60}$ and TCNQ via SSIM across a range of threshold ratios and variances (Fig. 9). We see that SSIM falls off for TCNQ at low threshold ratios for all variances $\sigma$, and in the limit of low $\sigma$ and threshold ratio-a trend consistent for both sampling patterns. This behavior is expected, as reducing threshold ratio and decreasing $\sigma$ are both tantamount to applying an increasingly strong low-pass filter [Fig. 8(d)]. Surprisingly, the filtering at low $\sigma$ and threshold ratio produces distinctly higher SSIM for the defect compared to the global image, though visual inspection revels intense lattice warping. In contrast to TCNQ, which has similar trends in performance for both patterns, $\mathrm{C}_{60}$ is quite different. For Lissajous, the SSIM falls off at threshold ratios around 20\% independently of $\sigma$. The rotated line maintains high SSIM at low $\sigma$, though a transition line develops with increasing $\sigma$ that exponentially confines good SSIM to low threshold ratios. While the global diagrams for $\mathrm{C}_{60}$ are seemingly immune from SSIM degradation at low threshold ratio, the defect diagrams show a slight dip. Visual inspection of reconstructions in this regime reveals heavy and unsatisfactory smoothing which retains a resemblance of the step defect and an accordingly high SSIM. For the samples and patterns shown in Fig. 9 though, overlapping high-SSIM regions across global and defect diagrams provide guidance on the optimal parameter space for defect-lattice reconstruction and provide proof 

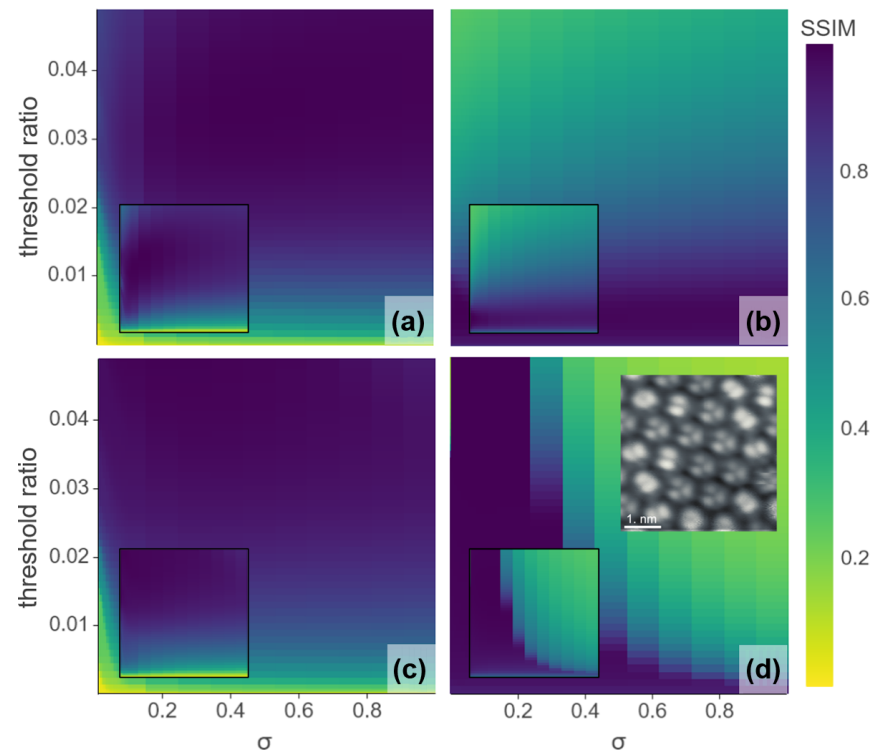

FIG. 9. SSIM evaluated for CS reconstructions of TCNQ [(a), (c)] and $\mathrm{C}_{60}[(\mathrm{~b}),(\mathrm{d})]$ across varying levels of $\sigma$ (the width of the variance in the DCT weight model) and threshold ratio (the relative number of of nonzero coefficients used by the reconstruction algorithm). The top and bottom rows, respectively, correspond to reconstructions performed using Lissajous and rotated line sampling patterns. All reconstructions performed with sampling density $\rho=0.2$.

of principle for effectively tuning the thresholding function parameters.

To better understand the rather unexpected behavior of $\mathrm{C}_{60}$, we refer back to its DCT [Fig. 2(c)]. TCNQ and FeSe exhibit compact density at low frequencies (upper left corner) in DCT [Figs. 2(a) and 2(b)], with clear peaks corresponding to ordered structure. For $\mathrm{C}_{60}$, the DCT is not only more spread out at low frequencies but also reveals two vertical broad bands of finite intensity. By filtering out the various parts of the DCT, we have verified that the bands are produced by a combination of the step in the middle of the image and randomized short-range orientations of individual $\mathrm{C}_{60}$ atoms [upper inset of Fig. 9(d)]. Indeed, it is well known that $\mathrm{C}_{60}$ films are orientationally disordered due to multiple energy-equivalent orientations of the buckyball frame of the molecule [38]. Technically, the structure lacks long-range order in this case, which we postulate leads to complex frequency-domain interactions with sampling patterns and the thresholding function that compromise the performance of the algorithm, particularly at lower sampling density. As a corollary, the anomalies in CS reconstruction can potentially reveal interesting properties of the image, such as the lack of long-range order.

In our studies, SSIM proved to be a faithful reconstruction quality metric in terms of capturing the influence of unwanted artifacts. While MSE was able to capture general trends, the metric lacks SSIM's useful universal scale, making cross comparison of images and phase diagrams more difficult. Furthermore, MSE is not adept at capturing structural artifacts [39] and this flaw is displayed in reconstruction diagrams created using the metric (Fig. 7). While they moderately resemble those for SSIM (Fig. 5), these diagrams fail to properly differentiate between good reconstructions and those marred by artifacts. As a particularly harsh example, the poorly reconstructed image of TCNQ at noise intensity and sampling density both equal to 0.1 yields a poor SSIM but an MSE closer to average. At the same parameters for FeSe, the metrics show a reverse trend, though visual inspection of the reconstruction here yields largely intact long-scale structure and perturbed small-scale structure, i.e., the lattice and defects. This indicates that the right choice of quality metric may be dependent on the feature of interest.

\section{CONCLUSIONS}

Our results were attained by applying a simulated CS approach to previously acquired data sets. Based on these results, CS could be used dynamically with STM to dramatically reduce measurement time for a given image size or allow for a larger field of view if measurement time is held constant. This allows for more efficient sampling of materials, with greater extent and higher probability to locate regions of interest. We show lesser but good reconstruction for moderate noise which indicates the robustness and applicability of this method for real-world STM data collection-benefits which should readily extend to other scanning probe microscopies. The added noise has a functional equivalence to dwell time, and the effective noisy reconstruction suggests further speed-ups as a byproduct of shorter required dwell times. The outlined methodology is readily applicable to imaging of periodic structures, but also to defects and imperfections. It is clear that with proper thresholding initialization, satisfactory reconstruction can be obtained without the presence of sampling pattern artifacts. However, since the optimal parameters are sensitive to differences between images and sampling patterns, to properly set the weights it is advisable to inform the model with prior imaging of a similar sample. Future studies evaluating algorithm parameters across many images could help determine the effectiveness and consistency of leveraging prior knowledge for reconstruction.

We intentionally used a simple framework to set up a baseline on which future improvements in CS reconstruction can be made. It is important to note though, that CS is a highly extensible framework open to more intelligent and in situ approaches to determine the most effective sampling path and select successful algorithm parameters and transform matrices. Intriguingly, anomalies in CS reconstruction, such as the ones we observed with $\mathrm{C}_{60}$ may signal interesting properties of the material, such as the lack of long-range order or dynamic processes in the experiment, which can then be studied with higher fidelity. The code and data used and developed in this paper is available on Github [40].

\section{ACKNOWLEDGMENTS}

We gratefully acknowledge Seokmin Jeon and Simon Kelly for their help with sample preparation for STM experiments with adsorbed molecules. Data analysis and interpretation was sponsored by the U.S. Department of Energy, Office of Science, Basic Energy Sciences, Materials Sciences and Engineering Division. Experimental data was acquired at the Center for Nanophase Materials Sciences, which is 
a DOE Office of Science User Facility. Student (B.E.L., A.F.G.) research support was provided by the DOE Science Undergraduate Laboratory Internships (SULI) program. This research used resources of the Compute and Data Environment for Science (CADES) at Oak Ridge National Laboratory, and the paper has been coauthored by employees of UTBattelle, LLC, which are supported by the U.S. Department of Energy, Office of Science under Contract No. DE-AC05-
00OR22725. The U.S. Government retains and the publisher, by accepting the paper for publication, acknowledges that the U.S. Government retains a nonexclusive, paid-up, irrevocable, worldwide license to publish or reproduce the published form of this paper, or allow others to do so, for U.S. Government purposes. The Department of Energy will provide public access to these results of federally sponsored research in accordance with the DOE Public Access Plan [41].
[1] A. R. Schmidt, K. Fujita, E.-A. Kim, M. J. Lawler, H. Eisaki, S. Uchida, D.-H. Lee, and J. C. Davis, Electronic structure of the cuprate superconducting and pseudogap phases from spectroscopic imaging STM, New J. Phys. 13, 065014 (2011).

[2] O. Fischer, M. Kugler, I. Maggio-Aprile, C. Berthod, and C. Renner, Scanning tunneling spectroscopy of high-temperature superconductors, Rev. Mod. Phys. 79, 353 (2007).

[3] J. E. Hoffman, Spectroscopic scanning tunneling microscopy insights into Fe-based superconductors, Rep. Prog. Phys. 74, 124513 (2011).

[4] Y. Hasegawa and P. Avouris, Direct Observation of Standing Wave Formation at Surface Steps Using Scanning Tunneling Spectroscopy, Phys. Rev. Lett. 71, 1071 (1993).

[5] M. F. Crommie, C. P. Lutz, and D. M. Eigler, Imaging standing waves in a two-dimensional electron gas, Nature (London) $\mathbf{3 6 3}$, 524 (1993).

[6] W. Ko, C. Ma, G. D. Nguyen, M. Kolmer, and A.-P. Li, Atomicscale manipulation and in situ characterization with scanning tunneling microscopy, Adv. Funct. Mater. 29, 1903770 (2019).

[7] J. Oppliger and F. D. Natterer, Sparse sampling for fast quasiparticle-interference mapping, Phys. Rev. Research 2, 023117 (2020).

[8] M. Honma, K. Akiyama, M. Uemura, and S. Ikeda, Superresolution imaging with radio interferometry using sparse modeling, Publ. Astron. Soc. Jpn. 66, 95 (2014).

[9] K. Kazimierczuk and V. Y. Orekhov, Accelerated NMR spectroscopy by using compressed sensing, Angew. Chem., Int. Ed. 50, 5556 (2011).

[10] D. J. Holland, M. J. Bostock, L. F. Gladden, and D. Nietlispach, Fast multidimensional NMR spectroscopy using compressed sensing, Angew. Chem., Int. Ed. 50, 6548 (2011).

[11] E. M. Simmerman, H. H. Lu, A. M. Weiner, and J. M. Lukens, Efficient compressive and Bayesian characterization of biphoton frequency spectra, Opt. Lett. 45, 2886 (2020).

[12] B. J. Lawrie and R. C. Pooser, Toward real-time quantum imaging with a single pixel camera, Opt. Express 21, 7549 (2013).

[13] M. Lustig, D. Donoho, and J. M. Pauly, Sparse MRI: The application of compressed sensing for rapid MR imaging, Magn. Reson. Med. 58, 1182 (2007).

[14] M. Lustig, D. L. Donoho, J. M. Santos, and J. M. Pauly, Compressed sensing MRI, IEEE Signal Processing Mag. 25, 72 (2008).

[15] L. He and L. Carin, Exploiting structure in wavelet-based Bayesian compressive sensing, IEEE Trans. Signal Processing 57, 3488 (2009).

[16] C. S. Oxvig, T. Arildsen, and T. Larsen, Structure assisted compressed sensing reconstruction of undersampled AFM images, Ultramicroscopy 172, 1 (2017).
[17] K. P. Kelley, M. Ziatdinov, L. Collins, M. A. Susner, R. K. Vasudevan, N. Balke, S. V. Kalinin, and S. Jesse, Fast scanning probe microscopy via machine learning: Non-rectangular scans with compressed sensing and Gaussian process optimization, Small 16, 2002878 (2020).

[18] Y. Nakanishi-Ohno, M. Haze, Y. Yoshida, K. Hukushima, Y. Hasegawa, and M. Okada, Compressed sensing in scanning tunneling microscopy/spectroscopy for observation of quasiparticle interference, J. Phys. Soc. Jpn. 85, 093702 (2016).

[19] D. Huang, T. A. Webb, C.-L. Song, C.-Z. Chang, J. S. Moodera, E. Kaxiras, and J. E. Hoffman, Dumbbell defects in FeSe films: A scanning tunneling microscopy and first-principles investigation, Nano Lett. 16, 4224 (2016).

[20] J. F. Claerbout and F. Muir, Robust modeling with erratic data, Geophysics 38, 826 (1973).

[21] D. L. Donoho, Compressed sensing, IEEE Trans. Inf. Theory 52, 1289 (2006).

[22] E. J. Candes, J. Romberg, and T. Tao, Robust uncertainty principles: Exact signal reconstruction from highly incomplete frequency information, IEEE Trans. Inf. Theory 52, 489 (2006).

[23] E. J. Candes and M. B. Wakin, An introduction to compressive sampling, IEEE Signal Processing Mag. 25, 21 (2008).

[24] T. Arildsen, C. S. Oxvig, P. S. Pedersen, J. Østergaard, and T. Larsen, Reconstruction algorithms in undersampled AFM imaging, IEEE J. Selected Top. Signal Processing 10, 31 (2015).

[25] J. Romberg, Imaging via compressive sampling, IEEE Signal Processing Mag. 25, 14 (2008).

[26] T. L. Jensen, T. Arildsen, J. Østergaard, and T. Larsen, Reconstruction of undersampled atomic force microscopy images: Interpolation versus basis pursuit, in 2013 International Conference on Signal-Image Technology \& Internet-Based Systems, Kyoto, Japan (IEEE, 2013), pp. 130-135.

[27] H. S. Anderson, J. Ilic-Helms, B. Rohrer, J. Wheeler, and K. Larson, Sparse imaging for fast electron microscopy, Proc. SPIE 8657, 86570C (2013).

[28] G. K. Wallace, The JPEG still picture compression standard, IEEE Trans. Consumer Electron. 38, xviii (1992).

[29] A. Maleki and D. L. Donoho, Optimally tuned iterative reconstruction algorithms for compressed sensing, IEEE J. Selected Top. Signal Processing 4, 330 (2010).

[30] I. Daubechies, M. Defrise, and C. De Mol, An iterative thresholding algorithm for linear inverse problems with a sparsity constraint, Commun. Pure Appl. Math. 57, 1413 (2004).

[31] T. Blumensath and M. E. Davies, Iterative thresholding for sparse approximations, J. Fourier Analysis Appl. 14, 629 (2008). 
[32] D. Donoho, De-noising by soft-thresholding, IEEE Trans. Inf. Theory 41, 613 (1995).

[33] C. S. Oxvig, P. S. Pedersen, T. Arildsen, J. Østergaard, and T. Larsen, Magni: A Python package for compressive sampling and reconstruction of atomic force microscopy images, J. Open Res. Software 2, e29 (2014).

[34] X. Qu, W. Zhang, D. Guo, C. Cai, S. Cai, and Z. Chen, Iterative thresholding compressed sensing MRI based on contourlet transform, Inverse Probl. Sci. Eng. 18, 737 (2010).

[35] S. van der Walt, J. L. Schönberger, J. Nunez-Iglesias, F. Boulogne, J. D. Warner, N. Yager, E. Gouillart, T. Yu et al., scikit-image: Image processing in Python, PeerJ 2, e453 (2014).

[36] Z. Wang, A. C. Bovik, H. R. Sheikh, and E. P. Simoncelli, Image quality assessment: From error visibility to structural similarity, IEEE Trans. Image Processing 13, 600 (2004).

[37] J. Dan, X. Zhao, and S. J. Pennycook, A machine perspective of atomic defects in scanning transmission electron microscopy, InfoMat 1, 359 (2019).

[38] J. Leaf, A. Stannard, S. P. Jarvis, P. Moriarty, and J. L. Dunn, A combined Monte Carlo and Hückel theory simulation of orientational ordering in $\mathrm{C}_{60}$ assemblies, J. Phys. Chem. C 120, 8139 (2016).

[39] Z. Wang and A. C. Bovik, Mean squared error: Love it or leave it? A new look at signal fidelity measures, IEEE Signal Processing Mag. 26, 98 (2009).

[40] https://github.com/amplifilo/CS-STM

[41] http://energy.gov/downloads/doe-public-access-plan 